\title{
An improved ant system algorithm for maximizing system reliability in the compatible module
}

\author{
Mana Sopa $^{1}$, Niwat Angkawisittpan ${ }^{2}$ \\ ${ }^{1}$ Faculty of Engineering, Mahasarakham University, Thailand \\ ${ }^{2}$ Research Unit for Computational Electromagnetics and Optical Systems, Faculty of Engineering, \\ Mahasarakham University, Thailand
}

\section{Article Info \\ Article history: \\ Received Jun 27, 2018 \\ Revised Mar 26, 2019 \\ Accepted Apr 9, 2019 \\ Keywords: \\ Ant colony optimization \\ Ant system \\ Decision-making \\ Meta-heuristic \\ Reliability optimization}

\begin{abstract}
This paper presents an improved Ant System (AS) algorithm called AS2 Swap for solving one of the reliability optimization problems. The objective is to selection a compatible module in order to maximize the system reliability and subject to budget constraints. This problem is NP-hard and formulated as a binary integer-programming problem with a nonlinear objective function. The proposed algorithm is based on the original AS algorithm and the improvement, focused on choosing the feasible solutions, neighborhood search with Swap technique for each loop of finding the solution. The implementation was tested by the five groups of data sets from the existing meta-heuristic found in the literature. The computational results show that the proposed algorithm can find the global optimal solution and is more accurate for larger problems.
\end{abstract}

Copyright () 2019 Institute of Advanced Engineering and Science. All rights reserved.

\section{Corresponding Author:}

Niwat Angkawisittpan,

Research Unit for Computational Electromagnetics and Optical Systems,

Faculty of Engineering, Mahasarakham University,

Khamriang Sub-District, Kantarawichai District, Maha Sarakham, 44150, Thailand.

Email: niwat.a@msu.ac.th

\section{INTRODUCTION}

The reliability is a significant of design measure in system engineering and manufacturing industries such as telecommunication systems, optical system design, electric power systems and etc. The design of a hardware system must be involved compatible module choice among available component types based on many characteristics such as performance, reliability, weight, cost and other. The reliability of the system is an indicator that shows the quality of products. For example, reliability optimization concerns the system reliability maximization subjected to performance and cost constraints [1], the system reliability maximization subjected to cost with multiple component choices [2, 3] and other works concerned with determining the redundancy allocation problem for series and parallel-series [4-12]. Evaluating the reliability optimization of such research, that presents different algorithms used to solve the problems such as Genetic Algorithm (GA), Ant Colony Optimization (ACO), Particle Swarm Optimization (PSO), Bee Colony Optimization (BCO) and more, reveals that the improvement of the original algorithm can solve the problem better than the original. However, the problems of the reliability optimization still need to have some constraints so that the result is in accordance with a given global optimal as required by the objective function.

The work presented in this paper deals with the reliability optimization problem for a series system with multiple-choice and many variables for decision-making that haven't had much attention focused on them. This problem is NP-hard and formulated as a binary integer-programming problem with a nonlinear objective function $[13,14]$. Throughout the past, there have been theories and methods for the overcoming of problems. These methods have had a different approach, as follows: branch and bound algorithm [15], 
neural network based on a quantized Hopfield network [16], AS algorithm [13], ACO with fuzzy set [14] and in some cases, have been applied to this problem and the yield optimization by using the Bee Algorithm (BA) [17]

The ACO algorithm has been formalized into a meta-heuristic for combinatorial optimization problems [18] and has been successfully applied to solve other problems such as the traveling itinerary problem [19], data clustering [20], weighted voting for multiple classifier systems [21], features selection optimization [22] and motor speed controller designs [23]. The original ACO algorithm is known as AS [24]-[25] and was extended, enhanced and improved to versions of AS including [26], Elitist AS, Ant-Q, Ant Colony System (ACS), MMAS, AS_rank, ANTS, BWAS, and Hyper-Cube AS, among others.

This paper aims to present the new improved AS algorithm based on the original AS, to solve the reliability optimization problem for a series system with multiple-choice and budget constraints. The improvement focused on choosing the most feasible solutions and neighborhood search with Swap technique for each loop of finding the solution.

The remainder of the paper is organized as follows. In section 2, the problem is introduced and expressed as a binary integer-programming problem with a nonlinear objective function. In Section 3, we describe the proposed algorithm in more detail. The computational experiments and results are given in Section 4. Finally, conclusions are drawn in Section 5.

\section{PROBLEM FORMULATION}

Consider a series system of $n$ subsystem. For each subsystem, there are different compatible module available with varying costs and reliabilities. The objective is to selection a compatible module in order to maximize the overall of system reliability and subject to budget constraints. The notations of problem formulation are introduced first:

$n$ the number of subsystems

$N_{i}$ the number of compatible module available for the subsystems $i$

$C_{i j}$ the cost of a subsystem $i$ using the compatible module $j$

$R_{i j}$ the reliability of the subsystem $i$ when the compatible module $j$ is used

$R_{s y s}$ the overall of system reliability

$B$ the total available amount of budget

Define the decisions variables $X_{i j}$ (with $i=1,2, \ldots, n$ and $j=1,2, \ldots, N_{i}$ ) as follows:

$$
X_{i j}=\left\{\begin{array}{lc}
1 & \text { if the subsystems } i \text { uses the compatible module } j \\
0 & \text { otherwise }
\end{array}\right.
$$

Define the objective function in order to solve the reliability optimization problem for a series system formulated as a binary integer-programming problem with nonlinear objective function.

$$
\begin{aligned}
& \text { Maximize } R_{\text {sys }}=\prod_{i=1}^{n}\left(\sum_{j=1}^{N_{i}} X_{i j} R_{i j}\right) \\
& \text { Subject to } \sum_{i=1}^{n} \sum_{j=1}^{N_{i}} X_{i j} C_{i j} \leq B \\
& \sum_{j=1}^{N_{i}} X_{i j}=1, \quad \forall i=1,2, \ldots, n \\
& X_{i j}=\{0,1\}, \quad \forall i=1,2, \ldots, n \text { and } j=1,2, \ldots, N_{i}
\end{aligned}
$$

The terms of the (1): represents the budget constraint, $B$ is an integer; the terms of the (2): represents the multiple-choice constraint and the terms of the equation; the terms of (3): defines the variables that are used to make decisions. When the result of a solution satisfies all constraints, it is called a feasible solution; otherwise, it is called an infeasible solution. 


\section{DESCRIPTION OF THE PROPOSED APPROACH}

\subsection{Basic principles of ant system (AS) algorithm}

AS is the first of the ACO algorithms [27-28] and it was inspired by the foraging behavior of real ant colonies. Ants are capable of finding the shortest path from food sources to the nest without using visual cues. When ants are searching for food, they initially explore the area surrounding their nest in a random manner. As soon as an ant finds a food source, it evaluates it and carries some food back to the nest. During the return trip, the ant deposits a chemical substance creating something called a pheromone trail on the ground. Ants use the intensity of the pheromone trails to communicate the food source information with other ants. The pheromone deposited, the amount of which may depend on the quantity and quality of the food, guides other ants to the food source. As other ants make their way along the path, they also leave the path with pheromone trails. As more ants pass by, more pheromone is deposited on the path; the trail with richer and more intensive pheromone has a higher probability to be chosen by the ants that follow. This positive feedback loop helps the ants establish the shortest paths between their nest and food sources.

Initially, three different versions of AS were proposed. These were called Ant-density, Ant-quantity and Ant-cycle. Nowadays, when referring to AS, one actually refers to ant-cycle and the two other variants were abandoned because of their lower performance. The general algorithm for AS is illustrated in Figure 1.

\begin{tabular}{|c|c|}
\hline $\begin{array}{l}\text { STEP 1: } \\
\text { STEP 2: }\end{array}$ & $\begin{array}{l}\text { Initialize the pheromone trails and parameters } \\
\text { Iteration } \\
\text { Repeat for each ant } \\
\text { Solution construction using global pheromone trails } \\
\text { Update the global pheromone trails } \\
\text { Until stopping criteria }\end{array}$ \\
\hline
\end{tabular}

Figure 1. The general AS algorithm [29]

There are steps to work, which are as follows: the first step consists of the initialization of the pheromone trails and setting of the default parameters. In the second step, first of iteration each ant applies the initialization of the pheromone trails to build solutions. For the next iteration, each ant uses global pheromone trails to determine the probability, with the state transition rule, to build complete solutions for the problem. This process is repeated until the stopping criteria are true.

\subsection{The AS-2Swap algorithm}

To apply AS algorithm to a combinatorial optimization problem, it represents the problem using a graph $G=(N, E)$ where $N$ is the set of nodes and $E$ is the set of edges. Figure 2 shows the model of the routes of ants between the nest and the food source

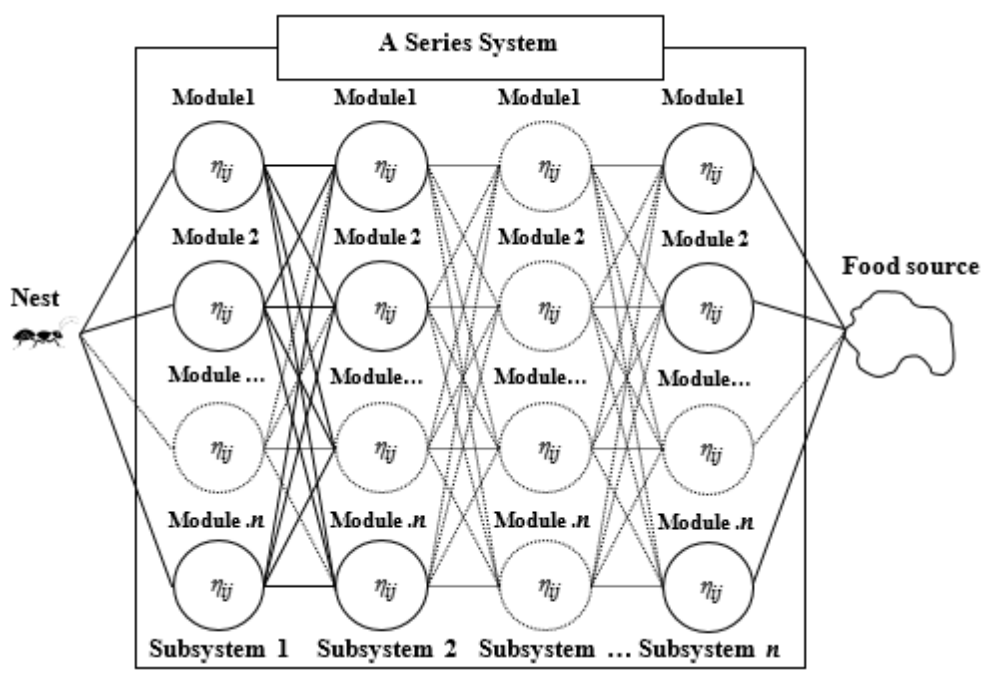

Figure 2. The model of the routes of ants between the nest and the food source 
When they are adapted to this problem, the set of $N$ represents the subsystems and the set of $E$ represents the available compatible module. This problem can be modeled as the routes between the nest and food source as shown in Figure 2. Flow chart of the improved AS algorithm is illustrated in Figure 3.

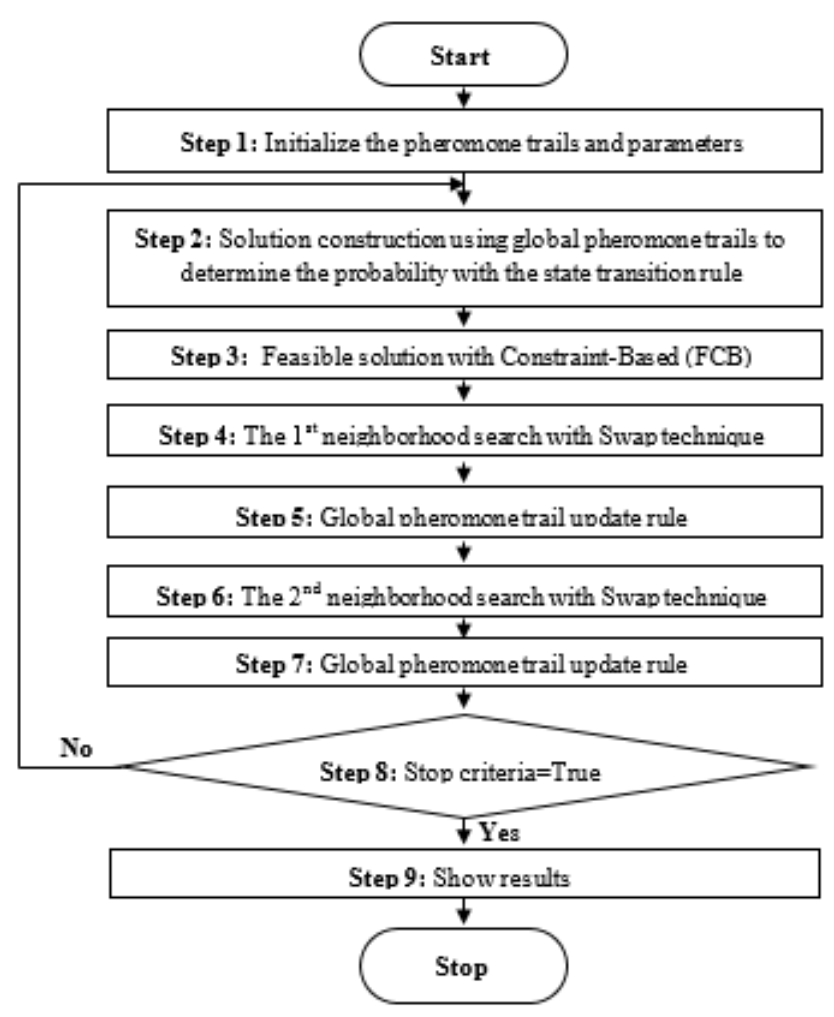

Figure 3. Flow chart of the AS-2Swap algorithm

The details of the proposed algorithm can be described in the following steps.

Step 1: Initialize sets, pheromone trails $\tau_{i j}(0)=\tau_{0}=0$, pheromone evaporation $\left(\Delta \tau_{i j}\right)=0$ and other parameters show that in Table 2 .

Step 2: Construct a set of solutions using global pheromone trails to determine the probability with the state transition rule. The state transition rule used by the AS algorithm is given in (4). This (4) represents the probability with which ant $m$ selects a compatible module $j$ for subsystem $i$ :

$$
P_{i j}^{k}(t)=\frac{\left[\tau_{i j}(t)\right]^{\alpha}\left[\eta_{i j}\right]^{\beta}}{\sum_{m=1}^{N_{i}}\left[\tau_{i m}(t)\right]^{\alpha}\left[\eta_{i m}\right]^{\beta}}
$$

Where $\tau$ is the intensity of pheromone; $\alpha, \beta$ are the parameters that controls $\tau_{i j}$ and $\eta_{i j} ; m$ is the total number of ants and $\eta_{i j}$ is the heuristics information between subsystem represented with $i$ and compatible module is represented with $j$. The problem specific heuristic information $\left(\eta_{i j}\right)$ is given in (5).

$$
\eta_{i j}=\frac{R_{i j}}{C_{i j}}
$$

Where $R_{i j}$ and $C_{i j}$ represent the meta-heuristics of reliability and cost. 
Step 3: Apply the feasible solution with constraint-based (FCB) algorithm, choose only feasible solutions under constraints from all ants in the colony build complete solutions. There are 3 sub steps to apply, as follows:

Step 3.1: Evaluate the reliability and cost of all solutions.

Step 3.2: Ranking and select only the sets of results that are feasible solutions.

Step 3.3: Select a feasible solution with the highest reliability value stored in the memory.

Step 4: Apply the first neighborhood search. A feasible solution with the highest reliability value from step 3 will be improved with one of local search techniques. This is performed based on the neighborhood search with Swap technique in order to find the better solution. To apply this work, it proceeds to change an in-turn pair of chosen compatible module by another pair in one turn. For each subsystem, the compatible module is indexed with their reliability. For example, the set of solution $S=\{a, b, c, \ldots\}$ indicates that subsystem 1 uses compatible module with index $a$, subsystem 2 uses compatible module with index $b$, subsystem 3 uses compatible module with index $c$, etc. Consider for example, a series system with 3 subsystems and 8 available compatible modules for each subsystem. By assuming that the set of solution in this loop are $S=\{4,1,6\}$ the Swap technique will evaluate the following solutions:

$$
\begin{aligned}
& S=\{3,1,6\} ; S=\{5,1,6\} ; S=\left\{4,1^{*}, 6\right\} ; \\
& S=\{4,2,6\} ; S=\{4,1,7\} ; S=\{4,1,5\} ;
\end{aligned}
$$

*Give the same result because it is minimum index or maximum.

All the solutions from the Swap technique are evaluated for the reliability and cost. Next step: select a feasible solution with the highest reliability value. If the reliability value is better than that from step 3 , it is stored as the best solution in the memory. Otherwise, it is returned using the reliability value from previous step.

Step 5: Apply the global pheromone trail update rule. In each loop, all ants in the colony have constructed their solutions with the global pheromone trail update rule the best solution from the latest step that is not able to guarantee that the best result or the global optimal is constructed. The amount of pheromone on each edge $(i, j)$ will be set to high value for the feasible solutions and set to low value for the infeasible solutions. This process is called the global pheromone trail update rule. The pheromone trail intensity is updated as follows.

$$
\tau_{i j}(t)=(1-\rho) \cdot \tau_{i j}(t-1)+\Delta \tau_{i j}
$$

Where $\rho$ a parameter between 0 and 1 , it represents the global pheromone trail evaporation. $\Delta \tau_{i j}$ is given in (7):

$$
\Delta \tau_{i j}=\sum_{k=1}^{m} \Delta \tau_{i j}^{k}
$$

Where $m$ is the number of ants and $\Delta \tau_{i j}^{k}$ is given in 8 :

$$
\Delta \tau_{i j}^{k}= \begin{cases}1 & \text { if } k^{t h} \text { ant choosescompatible module } j \text { for subsystemi } \\ 0 & \text { Otherwise }\end{cases}
$$

Step 6: Apply the second neighborhood search. A feasible solution from step 4 will be improved with the neighborhood search with Swap technique again. If the reliability value is better than that from step 4 , it is stored as the new best solution in the memory. Otherwise, return to the step 4 and do the process again.

Step 7: Apply the global pheromone trail update rule again for updating global pheromone trails.

Step 8: Stop criteria. The loops will be repeated until the stopping criteria are true. This is number of maximums the iteration value.

Step 9: Show results. 


\section{COMPUTATIONAL RESULTS}

\subsection{The data sample}

In order to evaluate the proposed approach, the data sets, presented in Tables 1, 4, 5 and 6 by Nahas \& Nourelfath in reference [13], consisting of 1) a series system with 15 subsystems with 60 and 100 variables, subject to budget constraints equal to $\$ 1000,2)$ a series system with 15 subsystems and 80 variables, subject to budget constraints equal to \$900,3) a series system with 25 subsystems and 166 variables, subject to budget constraints equal to $\$ 1400$ and 4) data set presented by Ahmadizar and Soltanpanah [14] consisting of a series system with 40 subsystems and 266 variables, subject to budget constraints equal to $\$ 2700$. The summary details of data samples are shown in Table 1 .

Table 1. Details of data samples

\begin{tabular}{cccc}
\hline Examples & Subsystems & Variables & Budget $(\$)$ \\
\hline 1 & 15 & 60 & 1000 \\
2 & 15 & 80 & 900 \\
3 & 15 & 100 & 1000 \\
4 & 25 & 166 & 1400 \\
5 & 40 & 266 & 2700 \\
\hline
\end{tabular}

\subsection{Test problems and results}

The test problem parameters are shown and specified in Table 2. From Table 2, all 10 trials run have been considered the number of all ants $=10$, maximum number of iterations $=1000, \rho=0.01, \alpha=0.8$ and $\beta=1$. The best results using the AS-2Swap algorithm and compare with AS, AS+ Local search [13] are illustrated in Figures 4(a-e).

Table 2. Parameters of test problems

\begin{tabular}{cc}
\hline Parameters & Number \\
\hline Number of all the ants & 10 \\
$\rho$ & 1000 \\
$\alpha$ & 0.01 \\
$\beta$ & 0.8 \\
Trial run & 1 \\
\hline
\end{tabular}

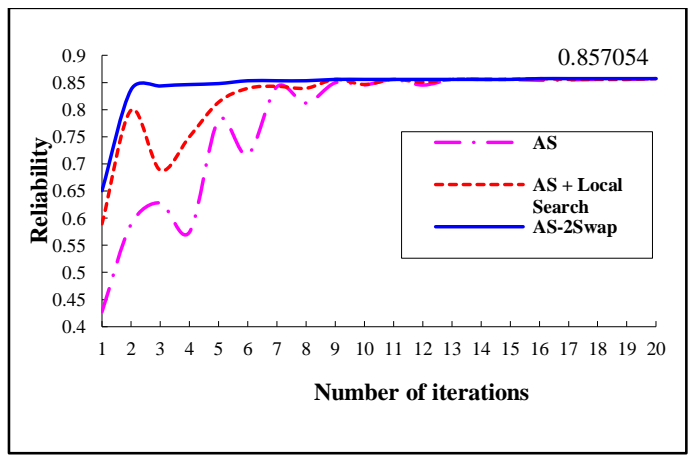

(a)

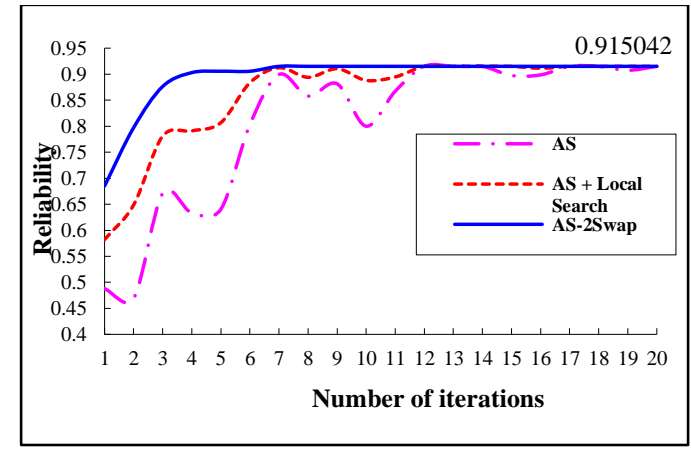

(b)

Figure 4. The results output of three algorithms for example 1-5 of data samples: (a) The results output of three algorithms for example 1, (b) The results output of three algorithms for example 2

The search space of example 1 is larger than $4.147 \times 10^{8}$. The AS-2Swap algorithm finds the global optimal was equal to 0.857054 with the number of iterations below 20 as shown in Figure 4(a). Selected compatible module are: 3-4-5-2-3-3-2-3-2-2-2-3-4-3-2. For the example 2, the search space is larger than $4.915 \times 10^{10}$. The best result found the global optimal was equal to 0.915042 with the number of iterations below 20 as shown in Figure 4(b). Selected compatible module are: 3-3-3-4-2-3-2-2-4-1-2-4-4-3-1. 


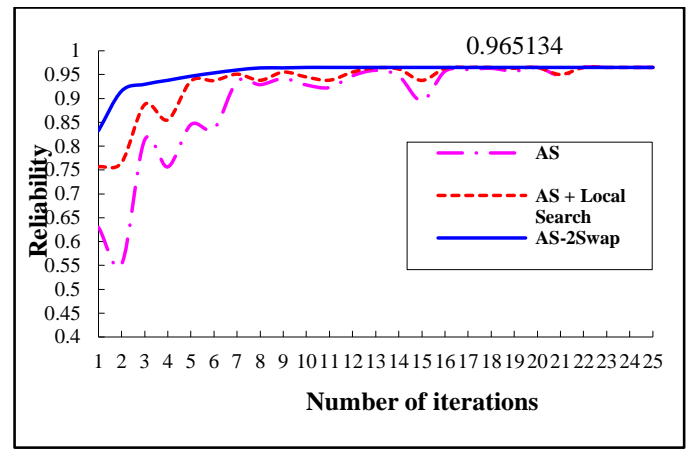

(c)

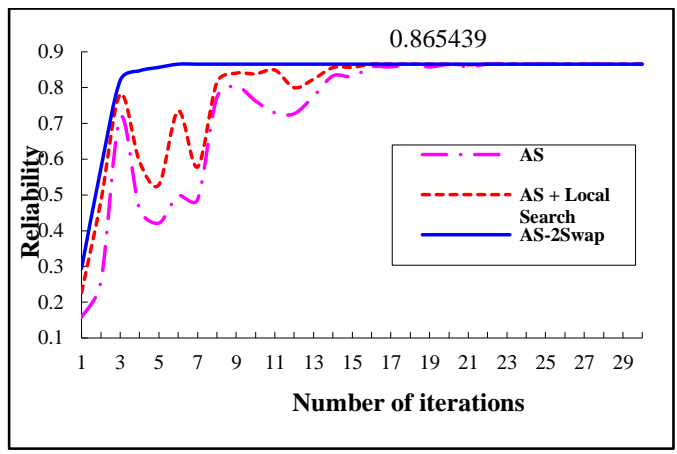

(d)

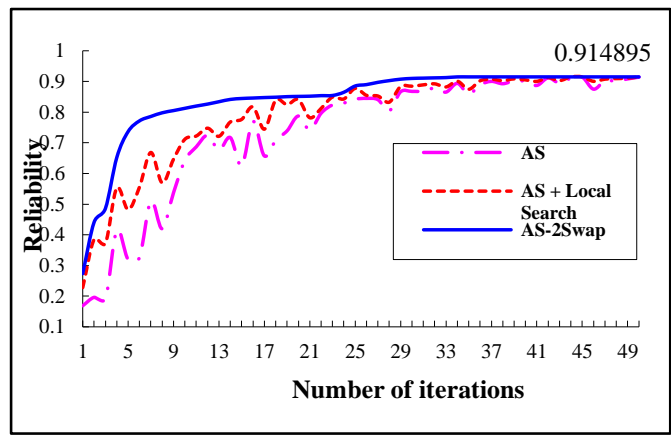

(e)

Figure 4. The results output of three algorithms for examples 1-5 of data samples: (c) The results output of three algorithms for example 3, (d) The results output of three algorithms for example 4,

(e) The results output of three algorithms for example 5

For example 3 , the search space is larger than $1.572 \times 10^{12}$. The best result found the global optimal was equal to 0.965134 with the number of iterations below 25 as shown in Figure 4(c). Selected compatible module are: 3-3-4-4-3-3-2-2-3-2-2-4-4-4-2. For example, 4, the search space is larger than $1.932 \times 10^{20}$. The best result found the global optimal was equal to 0.865439 with the number of iterations below 30 as shown in Figure 4(d). Selected compatible module are: 2-3-3-4-2-3-2-2-3-1-2-3-4-4-1-3-3-3-5-2-3-2-2-3-1.

The last example, example 5 has search space larger than $3.0399 \times 10^{32}$. The best result found the global optimal was equal to 0.914895 with the number of iterations below 50 as shown in Figure 4(e). Selected compatible module are: 3-3-4-4-3-3-3-2-3-2-2-4-4-4-2-3-3-4-4-3-3-3-2-3-2-3-3-4-4-3-4-3-3-3-2-24-4-4-2.

The summarized results of computations are shown in Table 3. For each subsystem, the results show the efficiency of the AS-2Swap in evaluating optimal reliability with the total cost under the budget constraints. As the results show, they have the highest and lowest reliability equal. The standard deviation is 0 , which demonstrates the effectiveness of this algorithm that can find the optimal reliability, precisely in all of the 10 trials run.

Table 3. The results of examples 1-5

\begin{tabular}{ccccccc}
\hline Sub systems & Variables & \multicolumn{4}{c}{ Reliability } & \multicolumn{2}{c}{ The best of } \\
& & Min. & Avg. & S.D. & Max. & total cost $(\$)$ \\
\hline 15 & 60 & 0.857054 & 0.857054 & 0 & 0.857054 & 990 \\
15 & 80 & 0.915042 & 0.915042 & 0 & 0.915042 & 895 \\
15 & 100 & 0.965134 & 0.965134 & 0 & 0.965134 & 995 \\
25 & 166 & 0.865439 & 0.865439 & 0 & 0.865439 & 1395 \\
40 & 266 & 0.914895 & 0.914895 & 0 & 0.914895 & 2695 \\
\hline
\end{tabular}


Finally, Table 4 gives a comparison between the results of AS with the improvement procedure [13] for examples 3 and 4 and ACS with the local search and fuzzy sets meta-heuristic information (Case 6) [14] for example 5. The proposed approach has been the same as ACS in examples 3 and 4, as it relates to the solutions but superior to AS in other areas. For example 5, the AS-2Swap has outperformed ACS and was able to get very good solutions for large problems at the computational time.

Table 4. The performance comparison

\begin{tabular}{cclcc}
\hline Subsystems & Variables & Methods & \multicolumn{2}{c}{ Reliability } \\
& & & Avg. & S.D. \\
\hline \multirow{2}{*}{15} & \multirow{3}{*}{100} & AS-2Swap & $\mathbf{0 . 9 6 5 1 3 4}$ & $\mathbf{0}$ \\
& & IAS & 0.96406 & 0.00050 \\
& & ACS & 0.965134 & 0 \\
25 & \multirow{2}{*}{166} & AS-2Swap & $\mathbf{0 . 8 6 5 4 3 9}$ & $\mathbf{0}$ \\
& & IAS & 0.86491 & 0.0038 \\
\multirow{2}{*}{40} & \multirow{2}{*}{266} & ACS & 0.865439 & 0 \\
& & AS-2Swap & $\mathbf{0 . 9 1 4 8 9 5}$ & $\mathbf{0}$ \\
& & ACS & 0.914794 & 0.000263 \\
\hline
\end{tabular}

\section{CONCLUSION}

This paper proposes a new efficiency algorithm based on the AS to solve the reliability optimization problem for a series system with multiple-choice and budget constraints. The proposed method is called the AS-2Swap which introduces two additional techniques in order to improve the search process included FCB algorithm and neighborhood search with Swap technique for each loop of finding the solutions. To show its efficiency, AS-2Swap was applied to test with five groups of data sets from the existing meta-heuristic available in the literature and compared with results from the recent approaches, i.e. AS with improvement procedure [13] and ACS with local search and fuzzy sets meta-heuristic information [14]. Computational results show that this algorithm can find the global optimal solution for decision-making and is more accurate for the large problems in computational time.

\section{ACKNOWLEDGEMENTS}

The authors would like to thank Research Unit for Computational Electromagnetics and Optical Systems, Faculty of Engineering, Mahasarakham University.

\section{REFERENCES}

[1] R. Meziane, Y. Massim, A. Zeblah, A. Ghoraf and R. Rahli, "Reliability optimization using ant colony algorithm under performance and cost constraints," Electric Power Systems Research, vol. 76, pp. 1-8, 2005.

[2] N. Ruan and X. Sun, "An exact algorithm for cost minimization in series reliability systems with multiple component choices," Applied Mathematics and Computation, vol. 181, pp. 732-741, 2006.

[3] H. Hashemi-dezaki and S.H. Hosseinian, "Optimized operation and maintenance costs to improve system reliability by decreasing the failure rate of distribution lines," Turk. J. Elec. Eng. \& Comp. Sci., vol. 21, pp. 2191-2204, 2013.

[4] R. Tavakkoli-Moghaddam, J. Safari and F. Sassanic, "Reliability optimization of series-parallel systems with a choice of redundancy strategies using a genetic algorithm," Reliability Engineering and System Safety, vol. 93, pp. 550-556, 2008.

[5] R. K. Gupta, A. K. Bhunia and D. Roy, "A GA based penalty function technique for solving constrained redundancy allocation problem of series system with interval valued reliability of components," Journal of Computational and Applied Mathematics, vol. 232, pp. 275-284, 2009.

[6] M. Agarwal and V. K. Sharma, "Ant colony approach to constrained redundancy optimization in binary systems," Applied Mathematical Modelling, vol. 34, pp. 992-1003, 2010.

[7] N. Beji, B. Jarboui, M. Eddaly and H. Chabchoub, "A Hybrid Particle Swarm Optimization Algorithm for the Redundancy Allocation Problem," Journal of Computational Science, vol. 1, pp. 159-167, 2010.

[8] V. K. Sharma, M. Agarwal and K. Sen., "Reliability evaluation and optimal design in heterogeneous multi-state series-parallel systems," Information Sciences, vol. 181, pp. 362-378, 2011.

[9] W. C. Yeh and T. Jung Hsieh, "Solving reliability redundancy allocation problems using an artificial bee colony algorithm," Computers \& Operations Research, vol. 38, pp. 1465-1473, 2011.

[10] H. Tekiner-Mogulkoc, D.W. Coit, "System Reliability Optimization Considering Uncertainty: Minimization of the Coefficient of Variation for Series-Parallel Systems," IEEE Transactions on Reliability, vol. 60, pp. 667-674, 2011.

[11] A. Chambari, et al., "bi-objective model to optimize reliability and cost of system with a choice of redundancy strategies," Computers \& Industrial Engineering, vol. 63, pp. 109-119, 2012.

[12] S.M. Miryousefi Aval and A. Ahadi, "Reliability Evaluation of Wind Turbine Systems' Components," Bulletin of Electrical Engineering and Informatics, vol. 5, no. 2, pp. 160-168, 2016. 
[13] N. Nahas and M. Nourelfath, "Ant system for reliability optimization of a series system with multiple-choice and budget constraints," Reliability Engineering and System Safety, vol. 87, pp 1-12, 2005.

[14] F. Ahmadizar and H. Soltanpanah, "Reliability optimization of a series system with multiple-choice and budget constraints using an efficient ant colony approach," Expert Systems with Applications, vol. 38, pp. 3640-3646, 2011.

[15] C.S. Sung and Y.K. Cho, "Reliability optimization of a series system with multiple-choice and budget constraints," European Journal of Operational Research, vol. 127, pp. 159-171, 2000.

[16] M. Nourelfath and N. Nahas, "Quantized hopfield networks for reliability optimization," Reliability Engineering and System Safety, vol. 81, pp. 191-196, 2003.

[17] W. Wongthatsanekorn and N. Matheekrieangkrai, "Bee Algorithm for Solving Yield Optimization Problem for Hard Disk Drive Component under Budget and Supplier's Rating Constraints and Hueristic Performance Comparison," Intelligent Automation and Systems Engineering, Lecture Notes in Electrical Engineering, vol. 103, pp. 203-216, 2011.

[18] M. Dorigo and G. Di Caro, "The Ant Colony Optimization meta-heuristic," in New Ideas in Optimization, D. Corne, M. Dorigo, F. Glover, Ed. London: McGraw-Hill, 1999, pp. 11-32.

[19] Z.K. Abdurahman Baizal, et al., "Generating Travel Itinerary Using Ant Collony Optimization," TELKOMNIKA (Telecommunication, Computing, Electronics and Control), vol. 16, no. 3, pp. 1208-1216, 2018.

[20] A. S. Girsang, T. W. Cenggoro and K.W. Huang. "Fast Ant Colony Optimization for Clustering," Indonesian Journal of Electrical Engineering and Computer Science (IJEECS), vol. 12, no. 1, pp. 78-86, 2018.

[21] A. Husin and K. R. Ku-Mahamud, "Ant System and Weighted Voting Method for Multiple Classifier Systems," International Journal of Electrical and Computer Engineering (IJECE), vol. 8, no. 6, pp. 4705-4712, 2018.

[22] Y. Hendrawan, et al., "Image Analysis using Color Co-occurrence Matrix Textural Features for Predicting Nitrogen Content in Spinach," TELKOMNIKA (Telecommunication, Computing, Electronics and Control), vol. 16, no. 6, pp. 2712-2724, 2018.

[23] D. Yadav, A. Verma, "Comperative Performance Analysis of PMSM Drive Using MPSO and ACO Techniques," International Journal of Power Electronics and Drive System (IJPEDS), vol. 9, no. 4, pp. 1510-1522, 2018.

[24] M. Dorigo, Optimization, "learning and natural algorithms," Ph.D. dissertation, Dipartimento di Elettronica, Politecnico di Milano, Italy, 1992.

[25] M. Dorigo, V. Maniezzo, and A. Colorni, "Ant System: Optimization by a colony of cooperating agents," IEEE Transactions on Systems, Man, and Cybernetics, Part B., vol. 26, pp. 29-41, 1996.

[26] M. Dorigo, M. Birattari and T. Stützle, "Ant Colony Optimization: Artificial Ants as a Computational Intelligence Technique," IEEE Computational Intelligence Magazine, vol. 1, pp. 28-39, 2006.

[27] M. Dorigo and L.M. Gambardella, "Ant colonies for the traveling salesman problem," BioSystems, vol. 43, pp. 73-81, 1997.

[28] M. Dorigo and L.M. Gambardella, "Ant Colony System: A cooperative learning approach to the traveling salesman problem," IEEE Transactions on Evolutionary Computation, vol. 1, pp. 53-66, 1997.

[29] C. Blum and M. Dorigo, "The hyper-cube framework for ant colony optimization," IEEE Transactions on Systems, Man, and Cybernetics Part B., vol. 34, pp. 1161-1172, 2004.

\section{BIOGRAPHIES OF AUTHORS}

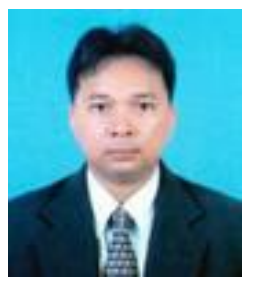

Mana Sopa was born in Surin Province, Thailand. He received his B.Sc.Ind.Ed. in Electronics and Telecommunication Engineering from Pathumwan Institute of Technology, Thailand in 2003, He received his M.S.Tech.Ed. in Computer Technology from the King Mongkut's Institute of Technology North Bangkok, Bangkok, Thailand in 2006. He is currently a Ph.D. student in Electrical and Computer Engineering at Mahasarakham University. His research interests include artificial intelligence and optimization technique.

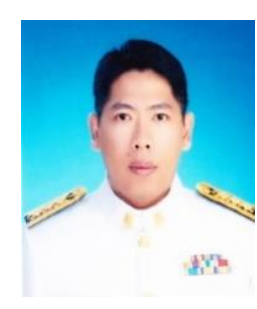

Niwat Angkawisittpan was born Khon Kaen, Thailand. He received his B.Eng. in Electrical Engineering with honors from Khon Kaen University, Thailand in 1997. He received his M.Sc. in Electrical \& Computer Engineering from Purdue University, Indiana, USA in 2003. Also, he received his Ph.D. in Electrical Engineering from University of Massachusetts Lowell, Massachusetts, USA in 2009. Since 2009, he has been with the Department of Electrical Engineering, Faculty of Engineering, Mahasarakham University, Maha Sarakham, Thailand as a Lecturer. He has authored or co-authored a number of papers in scientific journals and conference proceedings. His research interests include compact microstrip devices, Metamaterial applications for RF and microwave circuits, and electromagnetic material characterization. 